\title{
Comparison of Sialic Acids Excretion in Spot Urines and 24-Hour-Urines of Children and Adults
}

\author{
Susanne G. Fang-Kircher \\ Institute of Medical Chemistry, University of Vienna, Vienna, Austria \\ Dedicated to Professor Dr. E. Kaiser on the occasion of his 70th birthday
}

Summary: Sialic acids comprise all N- and O-acyl derivatives of neuraminic acid and are components of glycoproteins and glycolipids. Their concentrations vary physiologically with age but also in diseases such as inflammation, neoplastic tumours or in inborn genetic disorders causing abnormal sialic acid metabolism.

Determination of free and bound sialic acids in urine using the thiobarbituric acid method according to Warren (J Biol Chem 1959; 234:1971-5) was shown to be useful for the diagnosis of diseases that involve sialic acid metabolic disorders. This test - also used for the diagnosis of inborn errors of metabolic diseases, such as sialidosis, infantile sialic acid storage disease, Salla's disease, neuraminidase deficiency and others - should be included in the selective screening for storage diseases. With the reported number of mild, juvenile and adult forms of genetic disorders increasing, this diagnosis will also be useful for teenagers and adults. We therefore considered it important not to confine our investigation to children and compared the diagnostic value of 24-hour and spot urines.

As shown in 24-hour urines $(\mathrm{n}=242,128$ males, 114 females) the average excretion of sialic acids increases constantly during life, from $67.6 \mu \mathrm{mol}$ to $444.0 \mu \mathrm{mol}$ per day, as does the free $(27.5 \mu \mathrm{mol}$ to $217.1 \mu \mathrm{mol})$ and bound fraction $(40.1 \mu \mathrm{mol}$ to $226.9 \mu \mathrm{mol})$. The relative proportion of free and bound sialic acid shows only slight lifetime variations, the free fraction increases from about 40 percent the first few years to about 53 percent of total in the fifth decade. In the spot urines, the mean ratio of total free sialic acids and urinary creatinine $(\mathrm{mmol} / \mathrm{mol})$ decreases constantly during the first few decades, with a sharp drop during the first years of life (from 3 months -2 years: from 203.9 to 94.2 and 82.1 to 42.3 respectively; with 10 years: 52.3 and 22.4 respectively; in the sixth decade: 44.8 and 21.9). Similar findings could also be observed in the investigated 24-hour urines (correlation coefficient of ratios, $R=+0.981$ ).

The comparison of 24-hour urines and spot urines confirms the reliability of results for spot urines, however, the urine collection over an extended period yields additional information.

\section{Introduction}

Sialic acids, a group of $\mathrm{N}$ - and $\mathrm{O}$-acyl derivatives of neuraminic acid, are components of many complex carbohydrates of glycoproteins and glycolipids and occur in numerous tissues as demonstrated in various cell and organ cultures $(1-3)$. They are not only structural components of membranes but also of nuclei and cell organelles. Sialic acids are found in small amounts in many body fluids, such as serum, urine, saliva, semen, cerebrospinal fluid or pleural effusion $(5-7)$. Their amounts and biochemical composition vary in tissues, cells or fluids and with maturation or age $(8-10)$. Their concentrations usually change during diseases such as inflammation, diabetes mellitus, cardiovascular diseases, neoplastic tumours or in inborn genetic disorders causing sialic acid metabolic abnormalities $(1,2,4,5,11-15)$. In the numerous types of complex carbohydrates, the predominant form of sialic acids in humans is $\mathrm{N}$-acetyl- neuraminic acid (fig. 1), bound in alpha-glycosidic linkage to oligosaccharides, polysaccharides and glycoconjugates, glycoproteins or glycolipids; therefore a proteinor lipid-bound fraction can be discriminated from a free form (1).

Free and bound sialic acids can be determined by the thiobarbituric acid method of Warren (16). Possible in-

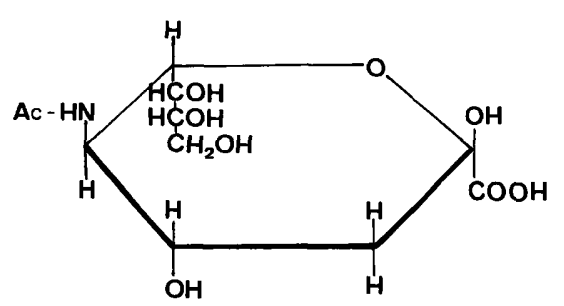

Fig. 1 N-Acetylneuraminic (D-glycero-D-galacto-5-amino-3,5deoxy-2-ketonone acid), the predominant form of sialic acid in humans (NeuAc, $\mathrm{C}_{11} \mathrm{H}_{19} \mathrm{NO}_{9}$, relative molecular mass $=309.3$ ). 
terferences from 2-deoxyribose are reported, but they can be eliminated by considering the substance specific absorption maximum $(549 \mathrm{~nm})$ and that of interfering sugars $(532 \mathrm{~nm})$. Special calculations of the absorption readings or slight test modifications improve the accuracy of this method $(4,7,16)$. However, the chemical determination of sialic acids based on Warren's method is simple and one of the most useful tests with sufficient specificity and high sensitivity (7). This method is also used for diagnosing sialic acid metabolic disorders and has been proposed to be included in the selective screening for inborn errors of metabolic diseases $(5,11,17$, 18).

The objectives of the present study were:

(i) to investigate the sialic acid concentrations and the sialic acid/creatinine ratios in 24-hour and spot urines in order to compare their predictiveness in differentiating between physiological and pathological conditions;

(ii) to obtain exact data on excreted free, bound and total sialic acids in 24-hour and spot urines during the course of life in order to identify any imbalances;

(iii) to learn about free, bound and total sialic acids not only in healthy children but also in healthy adults, as mild forms of juvenile and adult genetic disorders are being diagnosed in increasing numbers.

\section{Samples, Materials and Methods}

Samples

Twenty-four-hour urines and second morning urines of normal children, teenagers and adults $(n=176,90$ males, 86 females, aged $>2$ years - sixth decade) were collected at kindergartens, schools and university facilities. The urinary collection procedures in accordance with good clinical practice were explained to all volunteers and/or parents at study-entry. They were instructed to collect their second morning urine excretion following the 24-hour urine collection. Another 66 24-hour-urines and 14 spot urines were taken from children under 2 years of age ( 42 males, 38 females) who were hospitalized for reasons unrelated to sialic acid metabolism. The presence of pathological conditions particularly of the protein, blood, glucose, leukocytes and nitrite type were excluded by using a test strip (Combur 9-Test, Boehringer Mannheim, Mannheim, Germany). Urine samples were collected without preservative and stored at $-23^{\circ} \mathrm{C}$ until biochemical analysis.

Finally, urine samples of 13 patients suffering from rare genetic disorders were included in this study. They were discovered during urinary screening for metabolic diseases at the Institute of Medical Chemistry or were kindly provided by Dr. A. Cooper of the Willink Biochemical Genetics Unit of the Royal Manchester Children's Hospital, all of them with enzymatically established diagnosis. Urines from patients with the following diseases were investigated: infantile sialic acid storage disease $(n=1)$, sialidosis $(n=4)$, aspartylglucosaminuria $(n=1)$, mucopolysaccharidosis type IV Morquio's disease $(n=5)$ and fucosidosis $(n=2)$.

Materials and methods

All chemicals and reagents used were analytical-grade commercial products.

All absorbance readings were made using a Shimadzu Spectrophotometer UV-120-01 (Shimadzu Corporation, Kyoto, Japan).
Creatinine concentrations in the urine specimens were determined photometrically with a kinetic method based on the Jaffe reaction without deproteinization (Merckotest of Merck, Darmstadt, Germany).

Sialic acid levels were determined using the thiobarbituric acid assay based on Warren's method with slight modifications. In 100 $\mu \mathrm{l}$ aliquots of urine samples and standards ( $\mathrm{N}$-acetylneuraminic acid from Serva, Heidelberg, Germany), total sialic acid was evaluated after hydrolysis using $100 \mu 10.1 \mathrm{~mol} / \mathrm{l}$ sulfuric acid (Merck) for 60 minutes at $80^{\circ} \mathrm{C}$. Free sialic acid was investigated by addition of $100 \mu \mathrm{l}$ aqua bidest. instead of sulfuric acid under the same conditions. After incubation time, $100 \mu \mathrm{l}$ of $0.2 \mathrm{~mol} / \mathrm{l}$ sodium metaperiodate (Merck) in $9 \mathrm{~mol} / \mathrm{l}$ ortho-phosphoric acid (Merck) were added to all tubes, samples were mixed and incubated for an additional 25 minutes at room temperature. Then, $1 \mathrm{ml}$ of $100 \mathrm{~g} / \mathrm{l} \mathrm{so-}$ dium meta-arsenite (Sigma Chemical Corp., St. Louis, Missouri) in $0.5 \mathrm{~mol} / 1$ sodium sulphate (Merck) and $0.1 \mathrm{~mol} / 1$ sulphuric acid, and $3 \mathrm{ml} 6 \mathrm{~g} / 1$ of thiobarbituric acid (Merck) in $0.5 \mathrm{~mol} / 1$ sodium sulphate were added, samples were mixed, and heated for $15 \mathrm{~min}$ at $100{ }^{\circ} \mathrm{C}$ followed by a 5 minute ice-bath. After addition of $2 \mathrm{ml}$ cyclohexanone (Merck), the tubes were vigorously mixed and centrifuged $\left(7000 \mathrm{~g}\right.$ ) for 10 minutes at $2{ }^{\circ} \mathrm{C}$ (Hettich Rotina 48R, Tuttlingen, Germany). The absorbance of the cyclohexanone phase was measured immediately after centrifugation at two wavelengths $(532 \mathrm{~nm}$ and $549 \mathrm{~nm})$, and the calculations were performed according to Warren's method (13). Total and free sialic acids were calculated, the difference between these two being equal to the bound component.

\section{Accuracy, limits of detection, linearity and} imprecision

The recovery of total and free sialic acids in aqueous solution was measured over the concentration ranges of 3.233-1293.2 $\mu \mathrm{mol} / \mathrm{l}$, the correlation between the expected and measured concentrations was $R=1.00$ from $3.233-484.95 \mu \mathrm{mol} / 1$ and $R=0.998$ for concentrations from $484.95-1293.2 \mu \mathrm{mol} / 1$. The limit of detection was found to be about $3.233 \mu \mathrm{mol} / 1$, hence lower than the one reported by Lorentz et al. (7). The average recovery varied from $97-103 \%$, in concentrations lower than $13 \mu \mathrm{mol} / 1$ between 91 and $110 \%$.

A linearity of assay was given in concentrations up to $600 \mu \mathrm{mol} / \mathrm{l}$, higher concentrations were measured in diluted samples.

Imprecision data of sialic acid (concentration $=323.3 \mu \mathrm{mol} / \mathrm{l}$ ) were characterized by a coefficient of variation of $3.4 \%$ within-run $(n=10)$ or $4.9 \%$ on a daily basis $(n=20)$ for total sialic acid, and $2.9 \%$ or $4.1 \%$, respectively, for free sialic acid.

\section{Statistics}

All statistical calculations (means, standard deviations, correlations, coefficients of variation, analysis of variance (ANOVA), shape of distribution) were made using MICROSOFT ${ }^{\circledR}$. EXCEL and SAS/STAT ${ }^{\circledR}$. Due to the small numbers of probands of some age groups, tests for a sex effect and a shape of distributions were performed according to Romppanen et al. (14). In the larger groups ( $>13$ and more) there was evidence of a Gaussian distribution. For each age group, means and $1.96 \mathrm{SD}$ (2.5-97.5 percentiles) were calculated. The results in this study are comparable to the reference values reported for children and teenagers (for instance in 1 . c. $(11,15))$ and could be recommended as adequate.

\section{Results}

Age-related increase of urinary free, bound and total sialic acid in 24 -hour urines

In the 24-hour urines of healthy individuals, the average excretion of total, free and bound sialic acids increased significantly with increasing age (tab. 1). There was no evidence for sex-related differences. 
Tab. 1 Age-related excretion of total, free and bound sialic acid (means and 2.5-97.5 percentile intervals) in $\mu \mathrm{mol} / 24$ hours in col-

lected urines from healthy individuals from $3 / 12$ years to the 6th decade (21 different age groups).

\begin{tabular}{|c|c|c|c|c|c|c|}
\hline \multirow{3}{*}{$\begin{array}{l}\text { Age } \\
\text { (a) } \\
3 / 12 a\end{array}$} & \multirow{3}{*}{$\frac{\text { Number }}{24}$} & \multicolumn{5}{|l|}{ Sialic acid $(\mu \mathrm{mol} / 24 \mathrm{~h})$} \\
\hline & & Total $(\overline{\mathrm{x}} \pm 1.96 \mathrm{SD})$ & \multicolumn{2}{|c|}{ Free $(\overline{\mathrm{x}} \pm 1.96 \mathrm{SD})$} & \multicolumn{2}{|c|}{ Bound $(\overline{\mathrm{x}} \pm 1.96 \mathrm{SD})$} \\
\hline & & $67.6 \quad(28.4-106.8)$ & 27.5 & $(4.0-51.0)$ & 40.1 & $(12.1-68.1)$ \\
\hline $6 / 12 a$ & 15 & $91.7 \quad(38.2-145.2)$ & 38.9 & $(9.1-68.7)$ & 52.7 & $(20.6-84.8)$ \\
\hline $9 / 12 \mathrm{a}$ & 13 & $94.8 \quad(47.0-142.6)$ & 40.6 & $(12.2-69.0)$ & 54.1 & $(22.9-85.3)$ \\
\hline $1-<2 a$ & 14 & $95.6 \quad(43.5-147.7)$ & 38.4 & $(15.9-60.9)$ & 57.2 & $(17.2-97.2)$ \\
\hline $2 \mathrm{a}$ & 13 & $99.0 \quad(35.5-162.5)$ & 42.4 & $(8.6-76.2)$ & 56.6 & $(17.4-95.8)$ \\
\hline $3 a$ & 17 & $155.2(68.2-242.2)$ & 73.8 & $(34.6-113.0)$ & 81.4 & $(33.2-129.6)$ \\
\hline $4 a$ & 11 & $164.4(80.1-248.7)$ & 74.0 & $(20.7-127.3)$ & 90.4 & $(43.2-137.6)$ \\
\hline $5 a$ & 13 & $179.4 \quad(99.4-259.4)$ & 77.9 & $(26.4-129.4)$ & 101.4 & $(55.1-147.7)$ \\
\hline $6 a$ & 13 & $167.1 \quad(80.9-253.3)$ & 71.7 & $(31.3-112.1)$ & 95.4 & $(45.3-145.5)$ \\
\hline $7 a$ & 11 & $180.3 \quad(96.4-264.2)$ & 71.9 & $(27.6-116.2)$ & 108.4 & $(51.8-165.0)$ \\
\hline $8 \mathrm{a}$ & 5 & $208.3(116.9-299.7)$ & 84.4 & $(23.1-145.7)$ & 124.0 & $(72.9-175.2)$ \\
\hline $9 \mathrm{a}$ & 5 & $229.1(143.8-314.4)$ & 109.2 & $(44.4-174.0)$ & 119.9 & $(67.9-171.9)$ \\
\hline $10 \mathrm{a}$ & 7 & $240.0(116.3-363.7)$ & 105.7 & $(39.5-171.9)$ & 134.3 & $(54.7-213.9)$ \\
\hline $11-12 \mathrm{a}$ & 11 & $254.9(123.8-386.0)$ & 102.1 & $(39.0-165.2)$ & 152.8 & $(70.9-234.7)$ \\
\hline $13-15 \mathrm{a}$ & 9 & $271.1(109.8-432.4)$ & 123.3 & $(34.9-211.7)$ & 147.8 & $(60.0-235.6)$ \\
\hline $16-19 a$ & 8 & $316.1(144.6-487.6)$ & 148.1 & $(57.6-238.6)$ & 167.3 & $(93.0-241.6)$ \\
\hline $20-29 a$ & 15 & $346.2(177.6-514.8)$ & 148.1 & $(47.2-249.0)$ & 198.1 & $(91.4-304.8)$ \\
\hline $30-39 a$ & 18 & $354.5(197.9-511.1)$ & 157.1 & $(67.1-247.1)$ & 197.4 & $(112.0-282.8)$ \\
\hline $40-49 a$ & 9 & $336.6(173.1-500.1)$ & 150.0 & $(58.1-241.9)$ & 186.6 & $(65.1-308.1)$ \\
\hline $50-59 a$ & 5 & $322.5(168.9-476.1)$ & 172.1 & $(58.4-285.8)$ & 150.4 & $(97.2-203.6)$ \\
\hline $60-69 a$ & 6 & $444.0(349.0-539.0)$ & 217.1 & $(140.7-293.5)$ & 226.9 & $(186.2-267.6)$ \\
\hline
\end{tabular}

Age-related decrease of total and free sialic acid/creatinine ratios

Urinary creatinine is widely used to relate urine constituents to the glomerulum filtrate, especially in spot urines. Due to the increase in creatinine excretion during life, sialic acid/creatinine ratios show a decrease in all urine samples with increasing age, in spot urines as well as 24 -hour urines. As listed in table 2 (mean \pm 1.96 SD), a continuous age-related decrease of the total and free sialic acid/creatinine ratios in the spot urines could be observed. The highest ratios were found during the first months of life, with a sharp drop during the first 2 years, and a further decrease within the next 8 years. Values among adolescents and adults remained largely constant.

\section{Correlations between 24-hour urines and spot urines}

In order to compare the predictive diagnostic value of 24-hour and spot urines, 20 age groups including 243 probands (from $3 / 12$ years to 69 years, with the exception of $9 / 12$ years) were investigated. A satisfying inverse correlation between the means of the daily sialic acid excretion and the sialic acid/creatinine ratios of the spot urines could be observed ( $R=-0.804$, fig. 2a). The comparison of all age related means of the sialic acid/creatinine ratios in the spot urines, as well as collected urines, showed a very high correlation ( $R=+0.981$, fig. $2 b$ ).
The proportion (percentage) of free and bound sialic acids in various genetic disorders

Whereas free and bound sialic acid excretion show a constant increase over a lifetime, the relative proportion of free sialic acid changes only slightly. It is about $40 \%$

Tab. 2 Ratios of urinary sialic acid $\mathrm{mmol} / \mathrm{mol}$ creatinine in spot urines (means and 2.5-97.5 percentile intervals) from healthy individuals aged $3 / 12$ years to the sixth decade (20 different age groups).

\begin{tabular}{lrrrrr}
\hline $\begin{array}{l}\text { Age } \\
\text { (a) }\end{array}$ & $\begin{array}{l}\text { Num- } \\
\text { ber }\end{array}$ & \multicolumn{3}{l}{ Sialic acid (mmol/mol creatinine) } \\
\cline { 3 - 6 } & & \multicolumn{3}{c}{ Total $(\overline{\mathrm{x}} \pm 1.96 \mathrm{SD})$} & \multicolumn{2}{l}{ Free $(\overline{\mathrm{x}} \pm 1.96 \mathrm{SD})$} \\
\hline 3/12a & 4 & 203.9 & $(105.1-302.7)$ & 82.1 & $(24.3-139.9)$ \\
$6 / 12 \mathrm{a}$ & 3 & 152.0 & $(78.3-225.7)$ & 64.7 & $(18.6-110.8)$ \\
$1-<2 \mathrm{a}$ & 7 & 134.5 & $(67.5-201.5)$ & $55.5(16.9-94.1)$ \\
$2 \mathrm{a}$ & 13 & 94.2 & $(60.1-128.3)$ & $42.3(8.2-76.4)$ \\
$3 \mathrm{a}$ & 17 & 83.6 & $(47.5-119.7)$ & $41.0(13.0-69.0)$ \\
$4 \mathrm{a}$ & 11 & 84.9 & $(51.8-118.0)$ & $36.8(14.8-58.8)$ \\
$5 \mathrm{a}$ & 13 & 75.3 & $(45.7-104.9)$ & $30.4(11.2-49.6)$ \\
$6 \mathrm{a}$ & 13 & 70.0 & $(36.5-103.5)$ & $30.9(10.9-50.9)$ \\
$7 \mathrm{a}$ & 11 & 77.8 & $(41.1-114.5)$ & $31.8(10.4-53.2)$ \\
$8 \mathrm{a}$ & 5 & 54.4 & $(27.4-81.4)$ & $21.3(8.2-34.4)$ \\
$9 \mathrm{a}$ & 5 & 54.2 & $(27.0-81.4)$ & $26.3(12.0-40.6)$ \\
$10 \mathrm{a}$ & 7 & 52.3 & $(26.4-78.2)$ & $22.4(11.8-33.0)$ \\
$11-12 \mathrm{a}$ & 11 & 47.6 & $(26.6-68.6)$ & 18.7 & $(7.7-29.7)$ \\
$13-15 \mathrm{a}$ & 9 & 49.1 & $(26.6-71.6)$ & $22.6(10.1-35.1)$ \\
$16-19 \mathrm{a}$ & 8 & 39.6 & $(16.9-62.3)$ & 18.4 & $(9.0-27.8)$ \\
$20-29 \mathrm{a}$ & 15 & 27.7 & $(10.5-44.9)$ & 12.1 & $(3.9-20.3)$ \\
$30-39 \mathrm{a}$ & 18 & 38.3 & $(20.1-56.5)$ & $20.6(9.0-32.2)$ \\
$40-49 \mathrm{a}$ & 9 & 52.5 & $(28.8-76.2)$ & $22.6(11.0-34.2)$ \\
$50-59 \mathrm{a}$ & 5 & 46.6 & $(23.3-69.9)$ & $25.2(13.8-36.6)$ \\
$60-69 \mathrm{a}$ & 6 & 44.8 & $(23.4-66.2)$ & $21.9(12.9-30.9)$ \\
\hline
\end{tabular}




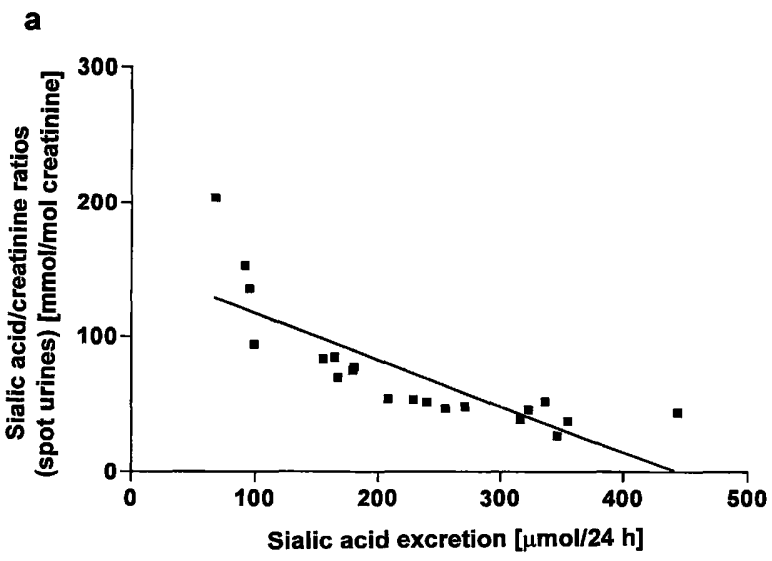

Fig. 2a Inverse correlation between the daily sialic acid excretion in 24 hour urines and the sialic acid/creatinine ratios in the spot urines (means of 20 different age groups, representing 243 probands).

Correlation coefficient: $R=-0.804(y=-0.34 x+150.99)$.

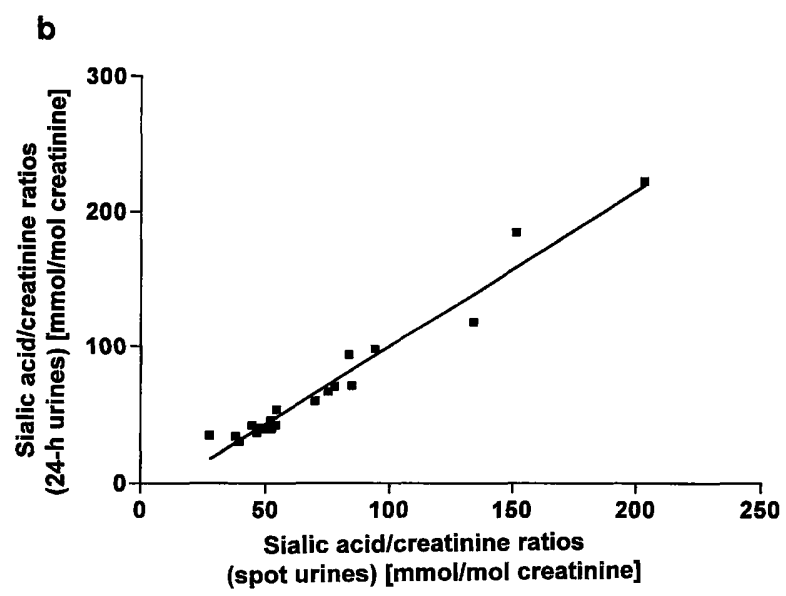

Fig. 2b Correlation between the sialic acid/creatinine ratio of spot urines and 24-hour urines (means of 20 different age groups, representing 243 probands). Correlation coefficient: $R=+0.981$ $(y=1.15 x-14.29)$.

in the 24-hour urines during the first years of life, with a slight increase at the age of 3 and in the fifth decade $(47.3 \%$ and $52.9 \%$ ) (fig. 3 , white bars). In the spot urines, the relative proportions of free sialic acid were slightly lower during the first few years and increased from $36.6 \%$ to $53.7 \%$ again in the fifth decade (fig. 3 , black bars).

Free sialic acid is elevated in disorders of sialic acid synthesis and transport, while bound sialic acid is increased in defects of several lysosomal enzymes (18, 19). As can also be seen from figure 3 , the proportion of free and bound sialic acids in some inborn metabolic diseases is clearly distinct from that in healthy individuals and can be used as a diagnostic criterion. Examples of pathological proportions in patients with various genetic diseases involving sialic acid metabolism are shown: infantile sialic acid storage disease $(86 \%)$, sialidosis (9\%), aspartylglucosaminuria (19\%), mucopolysaccharidosis type IV (Morquio's disease, 29\%) and fu- cosidosis: $8 \%$. With the exception of Morquio's disease, for all of these rare genetic disorders, the estimation of urinary sialic acids is a well-established screening quantity for the diagnosis. However, the investigation of total, free and bound sialic acids can also be helpful in the diagnosis of Salla's disease, galactosialidosis, neuraminidase deficiency, sialuria, mucolipidosis II and III, mannosidosis, and also Sandhoff's, Pompe's or Wolman's disease $(2,3,5,11,12,17,19-21$ and our own observations).

\section{Discussion}

Determination of urinary free, bound and total sialic acids is a useful method for diagnosing a number of inborn metabolic diseases $(17-21)$. Therefore it is essential to know about any age-dependent variations in their excretion in health and inborn metabolic disorders, as changes due to sialic acid metabolic diseases might be discovered at an earlier stage, before the patients are presenting with the fully developed clinical symptoms.

Usually genetic diseases are expected to be diagnosed in early childhood, and therefore some authors have focused on sialic acid excretion during the first years of life $(5,11,18)$. Mild juvenile and adult forms of genetic disorders are being increasingly reported as biochemical, enzymatic and molecular investigations improve the efficiency of diagnosis (22-24). For those mildly affected patients, exact physiological data from later life

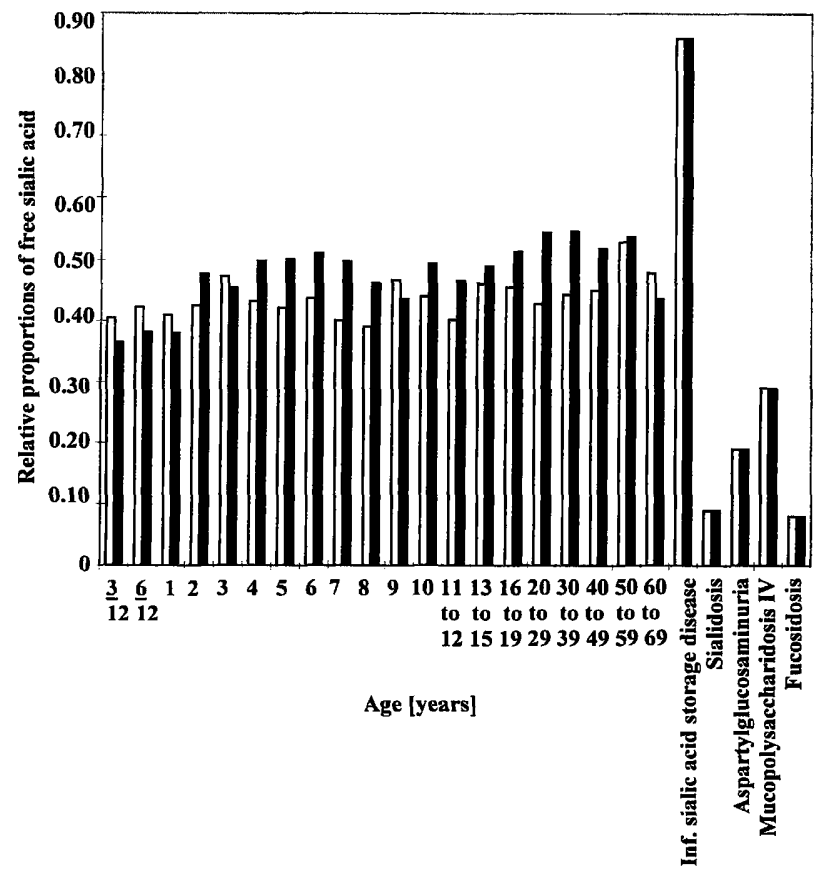

Fig. 3 Age-related relative proportions (means of percentages) of free sialic acid in 24-hour urines (white bars) and spot urines (black bars) from healthy individuals and patients with inborn genetic diseases. The results from patients with genetic metabolic diseases originate from 24-hour urines and/or spot urines. 
periods are needed in order to recognize any imbalances and to establish a diagnosis.

It is remarkable that in recent years several studies on serum and plasma sialic acid concentrations in elderly subjects were published in connection with arteriosclerosis, cardiovascular risks, diabetes mellitus and several malignancies. Urinary sialic acid concentrations in adults were mainly investigated in relation to diseases of the urinary tract $(10,25,26)$. Therefore, in this study it appeared necessary to also investigate urines from healthy adults.

The comparison of 24-hour and spot urines in this study confirms the reliability of results for spot urines, an observation reported by Waters et al., but without detailed age-related data (18). In our investigations we could show that there was a very high correlation between the age-related sialic acid/creatinine ratios of spot and 24hour urines, giving them the same diagnostic value. The relative proportions of free and bound sialic acids were also very similar in the different types of urine samples, a fact which allows a screening for metabolic disorders involving sialic acids in both types of urine specimen. Particularly in a pilot screening study it might be more practical to test spot urines from patients who are not toilet-trained. However, the collection over an extended period, for instance 24 hours, yields additional information about the sialic acid excretion and therefore appears preferable.

A significant agreement of the reference intervals in 24hour and morning or day/night urines has been observed by previous reports focusing on other urine metabolites $(27,28)$. The scatter of the excretory values was generally described as lower in 24-hour urines than in single urine samples, an observation we could not confirm, perhaps due to the different numbers of urines investigated in our study, $(n=176)$ versus 80 and 12 reported probands $(27,28)$.

Although the amount of total excreted urinary sialic acids increases significantly during life, serum concentrations only increase during the first months and remain stable over decades to come (reported reference ranges: $1312.6 \pm 161.7 \mu \mathrm{mol} / 1$ in children below 6 months; $2188.7 \pm 258.6 \mu \mathrm{mol} / \mathrm{l}$ in children and teenagers; $1694.1-2641.4 \mu \mathrm{mol} / \mathrm{l}$ in female adults; $1574.5-2625.2$ $\mu \mathrm{mol} / 1$ in males) $(7,9,25,29)$. Seppala et al. reported

\section{References}

1. Schauer R. Chemistry, metabolism and biological functions of sialic acids. Adv Carbohydr Chem Biochem 1982; 40:131234.

2. Thomas GH, Scocca J, Libert J, Vamos E, Miller CS, Reynolds LW. Alterations in cultured fibroblasts of sibs with an infantile form of a free (unbound) sialic acid storage disorder. Pediatr Res 1983; 17:307-12. that free sialic acid clearance varies directly with the creatinine clearance, indicating that this substance is filtered by renal glomeruli but is not reabsorbed by human kidney epithelial cells (20). This observation cannot be explained by changes in the glomerular filtration rate alone, as this rate is stable for many years and only decreases physiologically in elderly subjects. Part of the excreted sialic acids have to be drawn from the kidney cells themselves, or from cells of the urinary tract. Various types of kidney cells have been reported to synthesize free and bound sialic acids $(1,8)$. Roth et al. investigated the expression of polysialic acids in developing nephrons of rat kidneys with regional and transient differences (8). Similar differences in the expression of bound and free sialic acids were observed in ongoing studies on human renal epithelial cell cultures of papillary and cortical regions (in preparation).

\section{Conclusion}

In conclusion, our data show significant changes in urinary excretion of free, bound and total sialic acids in the course of life. Whereas there are increases in all three quantities that correlate with age, the relative proportions of free and bound sialic acids do not change significantly in healthly individuals of any age group and are distinct from those obtained with samples from patients with sialic acid metabolic disorders. The ratios of excreted urinary sialic acid and creatinine change markedly with age which could be shown in spot urines as well as 24-hour urines, they were therefore demonstrated to have the same diagnostic value.

It is still unclear whether glomerular filtration, kidney differentiation or development are responsible for the age-related urinary sialic acid excretion pattern, or whether additional physiological phenomena account for these differences. With this study, urinary sialic acid values for healthy adults could also be established, which might serve as a reference for diagnosing abnormal sialic acid metabolism even in adolescence or adulthood.

\section{Acknowledgements}

This work was supported by the "Forschungsgesellschaft für Mukopolysaccharidosen und ähnliche Erkrankungen". The author wishes to thank Dr. Barbara Scheider (Institute for Medical Statistics, University of Vienna, Head: Prof. Dr. P. Bauer) for her helpful assistance in statistics.
3. Seppala R, Titze F, Krasnewich D, Weiss P, Ashwell G, Barsh $\mathrm{G}$, et al. Sialic acid metabolism in Sialuria fibroblasts. J Biol Chem 1991; 266 (12):7456-61.

4. Roboz J, Suttajit M, Bekesi JG. Elimination of 2-deoxyribose interference in the thiobarbituric acid determination of $\mathrm{N}$-acetylneuraminic acid in tumor cells by $\mathrm{pH}$-dependent extraction with cyclohexanone. Anal Biochem 1981; 110:380-8. 
5. Hommes FA, editor. Determination of bound and free sialic acid in urine. In: Techniques in diagnostic human biochemical genetics: a laboratory manual. New York: Wiley-Liss Inc 1991:233-7.

6. Saifer A, Gerstenfeld S. Photometric determination of sialic acids in serum and cerebrospinal fluid with the thiobarbituric acid method. Clin Chim Acta 1962; 7:467-75.

7. Lorentz K, Weiß T, Kraas E. Sialic acid in human serum and cerebrospinal fluid. Comparison of methods and reference values. J Clin Chem Clin Biochem 1986; 24:189-98.

8. Roth J, Taatjes DJ, Bitter-Suermann D, Finne J. Polysialic acid units are spatially and temporally expressed in developing postnatal rat kidney. Proc Natl Acad Sci USA 1987; 84:1969-73.

9. Seider A, Graf N, Sitzmann FC. Wertigkeit der Sialinsäurebestimmung im Serum bei Kindern. Paed Paed 1992; 27:43-6.

10. Crook MA, Treloar A, Haq M, Tutt P. Serum total sialic acid and acute phase proteins in elderly subjects. Eur J Clin Chem Clin Biochem 1994; 32:745-7.

11. Cardo PP, Lombardo C, Gatti R. A simple detection of sialic acid storage disorders by urinary 'free' and 'total' sialic acid determinations. Clin Chim Acta 1985; 150:129-35.

12. Pelt JV, Dorland L, Duran M, Hokke CH, Kamerling JP, Vliegenthart FG. Sialyl- $\alpha 2-6-$ mannosyl- $\beta 1-4-\mathrm{N}$-acetylglucosamine, a novel compound occurring in urine of patients with $\beta$ mannosidosis. J Biol Chem 1990; 265 (32):19685-9.

13. Ozben T, Nacitarhan $S$, Tuncer N. Plasma and urine sialic acid in non-insulin dependent diabetes mellitus. Ann Clin Biochem $1995 ; 32: 303-6$.

14. Romppanen J, Mononen I. Age-related reference values for urinary excretion of sialic acid and deoxysialic acid: application to diagnosis of storage disorders of free sialic acid. Clin Chem 1995; 41:544-7.

15. Crook MA, Earle K, Morocutti A, Yip J, Viberti G, Pickup JC. Serum sialic acid, a risk factor for cardiovascular disease, is increased in IDDM patients with microalbuminuria and clinical proteinuria. Diabetes Care 1994; 17:305-10.

16. Warren L. The thiobarbituric acid assay of sialic acids. J Biol Chem 1959; 234 (8):1971-5.

17. Sewell AC. Urinary screening for disorders of heteroglycan metabolism. Results of 10 years experience with a comprehensive system. Klin Wochenschr 1988; 66:58-53.

18. Waters PJ, Lewry EM, Brimble AP, Pennock CA. Factors affecting the assay of urinary free and bound sialic acid. Biochem Soc Transactions 1992; 20:366S.
20. Seppala R, Renlund M, Bernardini I, Tietze F, Gahl WA. Renal handling of free sialic acid in normal humans and patients with Salla disease or renal disease. Lab Invest 1990; 63 (2):197203.

21. Pelt JV, Kamerling JP, Bakker HD, Vliegenthart JFG. A comparative study of sialyloligosaccharides isolated from sialidosis and galactosialidosis urine. J Inher Metab Dis 1991; 14:73040.

19. Scriver CR, Beaudet AL, Sly WS, Valle D. The metabolic and molecular bases of inherited disease. 7th ed New York: McGraw-Hill, Inc., 1995.

22. Palo J, Rauvala H, Finne J, Haltia M, Palmgren K. Hyperexcretion of free $\mathrm{N}$-acetylneuraminic acid - a novel type of sialuria. Clin Chim Acta 1985; 145:237-42.

23. Harzer K, Cantz M, Sewell AC, Dhareshwar SS, Roggendorf W, Heckl RW, et al. Normomorphic sialidosis in two female adults with severe neurologic disease and without sialyl oligosacchariduria. Hum Genet 1986; 74:209-14.

24. Chitayat D, Applegarth DA, Lewis J, Dimmick JE, McCormick AQ, Hall JG. Juvenile galactosialidosis in a white male: a new variant. Am J Med Gen 1988; 31:887-901.

25. Özben T. Elevated serum and urine sialic acid levels in renal diseases. Ann Clin Biochem 1991; 28:44-8.

26. Asami T, Tanaka A, Gunji T, Sakai E. Elevated serum and urine sialic acid levels in renal diseases of childhood. Clin Nephrol 1985; 23 (3):112-9.

27. Krieg M, Gunßer KJ, Steinhagen-Thiessen E, Becker H. Vergleichende quantitative Analytik klinisch-chemischer Kenngrößen im 24-Stunden-Urin und Morgenurin. Eur J Clin Chem Clin Biochem 1986; 24:863-9.

28. Khalaf AN, Böcker J, Kerp L, Petersen KG. Urine screening in outdoor volunteers: day versus night versus 24 hour collection. Eur J Clin Chem Clin Biochem 1991; 29:185-8.

29. Gressner AM, Henn KH. Evaluation of a fully mechanized enzymatic kinetic determination of sialic acid. J Clin Chem Clin Biochem 1985; 23:781-5.

\section{Received June 24/October 9, 1996}

Corresponding author: Dr. Susanne G. Fang-Kircher, Institute of Medical Chemistry, University of Vienna, Währinger Strasse 10, A-1090 Wien, Austria 